\title{
Popularisierung im (fachsprachlichen) Translationsunterricht - ein Aspekt der Translationsdidaktik
}

\author{
Cornelia Feyrer \\ Institut für Translationswissenschaft \\ Leopold-Franzens-Universität Innsbruck
}

This paper presents some approaches to and results of working with and integrating various text types of popular medical texts, as experienced when teaching translation. The premise for this work is the concept that popularized texts are still technical texts and can therefore be horizontally diversified and vertically stratified depending on their technical level. This, in combination with the practical framework set by the task and the persons involved in the interaction, makes various demands on the translator, who is called upon not only to provide a transcultural and inter-language translation of a technical text, but also to respect the specific circumstances of the target public at whom the target text is aimed. This is necessary for skoposoriented adaptation of the source material.

\section{Thematisches und Zielsetzungen}

Translationskompetenz umfaßt im Zeitalter der Globalisierung bzw. im Zeitalter der allumfassenden Informationsgesellschaft eine Vielzahl von Teilkompetenzen, zu welchen intra- und interkulturelles Informations- und Wissensmanagement genauso gehören wie kontrastive und v.a. inter- und transkulturelle Fach-, Sach- und Text(sorten)kompetenz. Dazu zählen auch Textoptimierung und kulturelle Anpassung. Aus dem Berufsbild für Übersetzer und Dolmetscher des BDÜ leitet Göpferich (2002:115) daher zurecht eine Tendenz im Anforderungsprofil an den Translator ab, die von textreproduzierenden Tätigkeiten hin zu textneuproduzierenden Tätigkeiten geht. In dieser Hinsicht hat die Beschäftigung mit Popularisierung viel zu bieten, da sie über den didaktisierten Umgang mit Fachwissen hinaus genau diese Kompetenz in der kreativen, funktionalen, zweckorientierten (lokalisierten oder nicht lokalisierten) Textproduktion schult. Man kann hier wohl von einer Teilmenge der Aufgaben eines modernen Translators (cf. ibid.) sprechen. Wie auch Nord (2002:17) feststellt, haben Studierende "für viele Textsorten nur eine passive, aber keine aktive Textsortenkompetenz". Durch das Popularisieren werden rezeptive und produktive Strategien der Arbeit mit Texten geübt, wodurch wiederum passive und aktive Textkompetenz erworben wird, die auch auf andere Textsorten übertragen werden kann. Popularisierende Texte ${ }^{1}$ haben sich daher sowohl im allgemein- als auch im fachsprachlichen Bereich aufgrund ihrer textsortenspezifischen wie auch pragmatisch-situativen Komplexität als wertvoll für die Schulung translationsrelevanter Teilkompetenzen erwiesen. Im Folgenden soll daher anhand 
von Beispielen aus dem Fachbereich Medizin exemplarisch aufgezeigt werden, was die Beschäftigung mit Popularisierung und dem popularisierenden Fachtext in der Translationsdidaktik mit Blick auf Text(sorten)spezifik, Pragmatik und Kultur leisten kann.

\section{Zum Aufgabenprofil des Popularisators bzw. popularisierenden Translators}

Die populärwissenschaftliche Aufbereitung von Fachwissen ist zum integralen Bestandteil unserer Kultur(en) und mit der zunehmenden Spezialisierung der Fachgebiete zu einer gesellschaftlichen Notwendigkeit geworden, die vor allem in universellen Wissenschaftsbereichen wie der Medizin - auch den Translator auf den Plan ruft und einen integrativen Bestandteil seiner Tätigkeit im Sinne eines Kommunikationsexperten ausmacht. Der Popularisator hat eine Brückenfunktion zwischen Fachwelt und Laienöffentlichkeit und muß es verstehen, komplizierte fachspezifische Inhalte für den Laien verständlich und konkret umsetzbar aufzubereiten und somit auf sprachlicher wie auch auf pragmatischer Ebene intra- wie auch intersprachlich und -kulturell einen Codewechsel zu vollziehen. Er muß Kompetenzen eines Fachmannes mit jenen eines Texters, der auf argumentativer, stilistischer, lexikalischer und vor allem pragmatisch-kultureller Ebene auf sein Zielpublikum und den Skopos seines Zieltextes eingehen kann, vereinen. (cf. Gläser 1990:174-178). Popularisierung stellt schon in der Einzelsprache komplexe Anforderungen an den Texter oder Textoptimierer, kommt die Dimension der Translation, des Sprach- und Kulturtransfers, hinzu, ergibt sich ein pluridimensionales Anforderungsprofil an die Arbeit des Kommunikationsexperten. Dies stellt auch für den Didaktiker im Unterricht eine Herausforderung dar. Der Popularisator - und noch in gesteigertem Maße der Translator, tritt er in Personalunion auch als Popularisator auf - wird zum Texter eines "parler pour les autres" (Authier 1982:44), also zum Sprachrohr in mehrfacher Hinsicht: Er spricht als Mediator für den Wissenschafter, den Fachmann, und er spricht gleichzeitig für sein Zielpublikum, indem er die zu übermittelnde Information an die Erfordernisse von Zieltext und -rezipienten anpaßt, hat also metakommunikative Funktion. Der popularisierende Translator hat so über seine Funktion als Sprach- und Kulturmittler hinaus die Funktion des Mediators, Kommentators und Didaktikers zu erfüllen, um seinem Leser zu ermöglichen, "d'y tracer un itinéraire personnel" (Jacobi 1984:51). Dieser itinéraire personnel impliziert jedoch auch, daß der popularisierte Text in der Regel einen für eine bestimmte Situation, einen spezifischen Kontext und eine bestimmte Rezipientensituation gemachten Text-in-Funktion bzw. Text-in-Situation darstellt und nicht generell mit wissenschaftlich-fachlicher Informationsvermittlung im Sinne der Vermittlung von Systemwissen gleichgesetzt werden kann (cf. Kroman \& Thomsen 1989:144) - ein Aspekt, der sich auf die entsprechende Didaktik auswirkt. 


\section{Zum Verhältnis von Fachsprache, Popularisierung und Transla- tion(sdidaktik)}

In den einzelnen Fachbereichen sind aufgrund situativer Umstände und terminologischer Differenzierung spezifische Fachsprachen, die innerhalb eines Faches unterschiedliche Fachlichkeitsgrade aufweisen, entstanden. Hoffmann ( $\left.{ }^{2} 1985: 58-79\right)$ spricht daher von horizontaler Gliederung, einer thematischen Differenzierung, und vertikaler Schichtung, einer Differenzierung nach dem jeweiligen Fachlichkeitsgrad. Aufgrund der starken interdisziplinären Vernetzung von Fachgebieten und der sowohl intra-, wie auch interfachlich und fachextern notwendigen Interaktion ist es oft schwierig, von einer autonomen Fachsprache eines bestimmten Fachgebietes zu sprechen, da diese Autonomie de facto meist weder inhaltlich noch sprachlich noch pragmatisch existiert (cf. Pöckl 1990:267). Es stellt sich somit auch die Frage, wo Popularisierung beginnt. In weiterem Sinne kann schon eine Interaktion zwischen zwei Fachleuten unterschiedlicher Teilfachgebiete desselben Faches Grundzüge der Popularisierung tragen und zumindest teilweise popularisierenden Charakter haben. Daher sprechen wir vom popularisierenden Fachtext, einer Menge von Texten mit einer gewissen Graduierung und jeweiligen Fokussierung auf Fach(sprach)lichkeit oder Popularisierung.

Was zur Grundkompetenz des popularisierenden Autors gehört, gehört erst recht zur Grundkompetenz des (popularisierenden) Translators bzw. des Translators popularisierender Texte. Allerdings sind die Anforderungen, mit denen sich der Translator konfrontiert sieht, und die auch in eine entsprechende Didaktik einfließen sollten, um die Komponenten des Sprach- und vor allem Kulturtransfers erweitert, in welche die Translation von fachinterner wie auch - externer Kommunikation eingebettet ist, wobei der Translator nicht selten zum Textoptimierer und -adaptierer wird (cf. Albin 1998:118). Er muß über eine kontrastive inter- bzw. transkulturelle Textsortenkompetenz als Teilkompetenz der Translationskompetenz verfügen. Daraus leitet sich auch eine fruchtbare Interaktion zwischen (Fach)Textlinguistik und Translationswissenschaft ab. Sieht man unter dem Postulat einer funktionalen Translationstheorie Fachkommunikation in ihrer ganzen Bandbreite an Fachlichkeitsgraden und unterschiedlichen Interaktionsrahmen als über das rein Fach(sprach)liche weit hinausreichendes, komplexes interaktanten- und situationsdeterminiertes Interaktionsgebilde, so wird der Handlungsrahmen, in welchem sich der Translator bewegen muß, über das rein (Fach)Sprachliche und Terminologische hinaus, um pragmatisch-kulturelle Dimensionen wie Auftragssituation und -interaktionspartner, Funktion, Zweck, Intention und Erwartung, Medium, Zielgruppen, usw. bereichert. Dies determiniert letztendlich die (Ziel)Textgestaltung. Popularisierung vereinigt nunmehr Charakteristika von Fachtexten und allgemeinsprachlichen Texten. Der popularisierende Text ist im Vergleich zum reinen Fachtext in der Regel dynamischer, emotionsgeladener, argumentativ ausgefeilter und verfügt über eine facettenreichere Sprache, was Kommunikationsstrategien, Argumentation und stilistische 
Merkmale anbelangt (cf. Pöckl 1990:269). Daher eignen sich popularisierende Texte sowohl von ihrer textuellen Spezifik der Makro- und Mikrostruktur, bzw. stilistischen Merkmalen und Textsortenkonventionen als auch von den pragmatisch-situativen Rahmenbedingungen her gesehen gut zur Schulung translationsrelevanter Textkompetenz.

\section{Medizinische Kommunikation}

\subsection{Interaktionsgebilde}

Die medizinische Kommunikation stellt als "Einheit von medizinischer Wissenschaft und gesellschaftlicher Institution Gesundheitswesen" (Wiese 1984:12) ein sehr komplexes Interaktionsgebilde dar, das sich durch eine Vielfalt von funktions- und situationsgebundenen Fachlichkeitsgraden auszeichnet. Zudem spielt, gerade was die Medizin anbelangt, die effiziente Kommunikation zwischen Fachmann und Laie eine bedeutende Rolle, wobei die betreffenden Interaktanten eine ganz und gar inhomogene Gruppe darstellen. Auch der Laie erwirbt in unserer Informationsgesellschaft Fachwissen, Fachwissensteile und terminologisches Wissen, das er in sein eigenes Handlungsrepertoire einbaut. Löning (1994:104) bezeichnet dies als "semiprofessionelle und pseudo-professionelle Kategorisierungen". Herausragendstes Charakteristikum der medizinischen Kommunikation ist jedoch, daß sie je nach Interaktanten- und Situationsspezifik ganz und gar unterschiedliche Ausdrucks- und Vertextungsformen bzw. Fachlichkeitsgrade umfassen kann. Der Bogen spannt sich über die einzelnen medizinischen Disziplinen, aber auch über die Diversifikation an Fachlichkeitsgraden von der innerfachlichen Interaktion zwischen Fachleuten desselben Fachgebietes bis hin zur fachexternen Interaktion mit dem Laien.

\subsection{Aspekte aus dem Berufsleben des Translators im Bereich Medizin}

AbsolventInnen der Ausbildungsstätten für Translation finden sich oft auf Stellen wieder, wo sie nicht nur als reiner Translator eingesetzt werden, sondern als Textbearbeiter und gerade im medizinischen Bereich auch als Popularisator. Pharmafirmen setzen z.B. Translatoren in der Sachbearbeitung ein, wo sie u.a. Fachinformationen und Schulungsunterlagen für Mitarbeiter ohne medizinische Ausbildung, Texte für Presseaussendungen, Informationsbroschüren, Beipacktexte, Besuchsdokumente, Posterpräsentationen für Tagungen u.v.m. aufzubereiten haben.

Schon diese nur willkürlich genannten Textsorten zeigen eine Graduierung an Fachlichkeits- und auch Popularisierungsgrad auf: Schulungsunterlagen dienen firmeninternem, meist nichtmedizinischem Personal und haben vor allem einen didaktischen Anspruch. Besuchsdokumente (cf. dazu Feyrer 2002) sind für Pharmareferenten bzw. Ärzte gedacht, die 
von einem Produkt überzeugt und darüber fachlich informiert werden müssen, haben also appellativen und informativen Charakter. Es handelt sich hier um fachinterne Kommunikation mit hohem Fachlichkeitsgrad, eingebettet in einen sehr komplexen Interaktionsrahmen. Informationsmaterial für die breite Öffentlichkeit, Presseaussendungen und Broschüren zählen wiederum zur fachexternen Kommunikation und haben niedereren Fachlichkeits- und dafür hohen Popularisierungsgrad. Posterpräsentationen für Tagungen, Studienergebnisse, wissenschaftliche Beiträge sind fachintern auf ein spezifisches - wenn auch hier wiederum eine gewisse Heterogenität aufweisendes - Fachpublikum ausgerichtet und tragen die Grundmerkmale wissenschaftlicher Texte. U. E. können daher auch nach dem Grad der Popularisierung Texte, d.h. genauer gesagt Texte-in-Funktion bzw. Texte-in-Situation, klassifiziert werden. Eine solche Graduierung nach Textsorten und unterschiedlichem Fachlichkeits- bzw. Popularisierungsgrad kann wiederum Grundlage für Text- und Fachlichkeitsprogression in der Didaktik sein bzw. eine Progression der Arbeitsaufträge gewährleisten.

\section{Textsorten der Popularisierung im Translationsunterricht}

Kommt neben der Aufbereitung fachlicher Inhalte $\mathrm{zu}$ den einzelnen Textsorten die Translation solcher Texte hinzu, muß neben der sprachenpaarspezifischen die kulturelle Dimension berücksichtigt werden. Die Grundlagen dafür müssen den Studierenden in der Ausbildung vermittelt werden, damit sie die Fähigkeit erlangen, sich, in der konkreten Berufssituation möglichst rasch und effizient auf die individuellen Anforderungen von Arbeitsplatz und entsprechendem Aufgabenprofil einstellen zu können. Hierfür sind popularisierende Texte gut geeignet, da sie allgemein translationsrelevante Problemstellungen mit fachsprachenspezifischen vereinen und die Studierenden sowohl vor generell-theoretische und -praktische Herausforderungen stellen, als auch den Erwerb von fachspezifischen Translationsstrategien und die Auseinandersetzung mit fachsprachenspezifischen Translationsproblemen ermöglichen.

Wie die kurz umrissenen Aspekte aus dem Alltag des Translators im Umfeld einer Pharmafirma gezeigt haben, ist das Arbeitsfeld von AbsolventInnen einer Übersetzer- und Dolmetscherausbildung komplex und erfordert ein Einarbeiten in die individuellen Bedingungen und Anforderungen des konkreten Arbeitsplatzes. Für die Ausbildung heißt dies, daß allgemeine Grundlagen geboten und verallgemeinerbare, d.h. in den Grundzügen auf individuelle Arbeitsverhältnisse anpaßbare, Kenntnisse und Kompetenzen vermittelt werden müssen. Vor allem sollte aufgrund der Komplexität der Fachbereiche das Bewußtsein für die ganze Bandbreite von Fachlichem, von der Popularisierung bis zum Fachtext mit hohem Fachlichkeitsgrad, vermittelt werden. Hierbei kommt der Popularisierung neben dem gesellschaftlichen und beruflich-translationsrelevanten auch ein translationsdidaktischer Stellenwert als Vorstufe zur Fachsprache bzw. als Fachtextsorte im Sinne des popularisierenden Fachtextes zu. Im Folgenden 
sollen anhand einiger konkreter Beispiele Ansätze zur Arbeit mit popularisierenden Texten, ihrer Textsortenspezifik und Pragmatik, im Translationsunterricht exemplarisch illustriert werden.

\subsection{Popularisierung im Unterricht zur Translatorischen Basiskompe- tenz}

\subsubsection{Arbeitsbedingungen}

Am Institut für Translationswissenschaft der Universität Innsbruck dienen die allgemeinen Übersetzungsübungen und Proseminare, die die Studierenden im dritten oder vierten Semester absolvieren, zur Vorbereitung auf Diplom- bzw. Fachprüfungen, in deren Rahmen auch eine Fachsprache gewählt wird. In diesen Lehrveranstaltungen (LVs) soll eine möglichst große Bandbreite an Texttypen und Textsorten bzw. Themenschwerpunkten praktisch und translationstheoretisch exemplarisch abgedeckt werden, um möglichst umfangreich Einblick in die verschiedenen Aspekte der konkreten Translationsarbeit, aber auch der theoretischen Auseinandersetzung damit geben zu können. Sowohl im sprachübergreifenden Proseminar als auch in den LVs zur Translatorischen Basiskompetenz lassen sich Grundlagen vermitteln, die auf die Fachsprache Medizin vorbereiten und z.B. die entsprechenden Textanalysekompetenzen und Kenntnisse über in diesem Bereich relevante Textsorten vermitteln, sodaß eine auf die weiterführenden Kurse ausgerichtete Text- und Fachlichkeitsgradprogression möglich wird. Dies sollte in Zusammenarbeit mit den LVs zum Training der muttersprachlichen passiven und aktiven Textkompetenz geschehen.

\subsubsection{Der Translator als Popularisator}

Die im Folgenden beschriebene Unterrichtseinheit fordert, als Vorstufe zum Fachübersetzen, den Translator als Popularisator. Den Studierenden wurde im Rahmen von sprachübergreifend ausgerichteten Proseminaren zu Textgestaltung und Übersetzung und zur populärwissenschaftlichen Vertextung - nach vorangegangener Analyse popularisierender Texte - Material bestehend aus Fachtexten unterschiedlicher Textsorten zur Verfügung gestellt, auf Grundlage dessen ein populärwissenschaftlicher Beitrag für die Rubrik Gesundheit-Moderne Medizin eines TV-Magazins erstellt werden sollte. Es sollte also ein Text für ein breites, heterogenes Zielpublikum bestehend aus Nicht-Fachleuten erstellt werden, die Basisinformationen über eine neue Errungenschaft der medizinischen Forschung vermittelt bekommen sollten, nämlich Forschungsergebnisse über den Zusammenhang der Aktivität des Bakteriums Helicobacter pylori mit dem Entstehen von Magengeschwüren. Dies hatte Anfang der 90er zur Entwicklung eines neuen Kombinationsantibiotikums, dem Helicocin, geführt. Als Informationsmaterial, aus dem der Text entstehen sollte, bekamen die Studierenden Auszüge aus einer 
- damals aktuellen - medizinischen Abhandlung zum Helicobacter, in der die Entdeckung, die Wirkungsweise und die vorliegenden Studien zu diesem Bakterium und eine Beschreibung der vom Helicobacter hervorgerufenen Krankheitsbilder und schließlich Magenkarzinome als sehr wahrscheinliche Folge einer langfristigen Infektion mit Helicobacter pylori sowie die entsprechenden therapeutischen Strategien bei Indikation zur Eradikation des Helicobacter beschrieben wurden. Dazu kam ein Auszug aus einer Projektstudie eines Gastroenterologen, in dem der Helicobacter als Erreger der chronisch aktiven Gastritis bzw. in Zusammenhang mit chronisch rezidivierendem peptischen Ulcus beschrieben und das entsprechende Antibiotikaschema vorgestellt wurde. Der Text lieferte vom Mediziner für Mediziner gestaltete, also fachinterne, Informationen mit wichtigen Anmerkungen für den Praktiker inklusive einer Kurzdarstellung zu den bestehenden Diagnoseverfahren. Dazu kam weiters eine Dokumentation zur entsprechenden Marketingstrategie sowie diesbezügliche weiterführende Erklärungen und die Beantwortung bzw. gemeinsame Recherche und Abklärung von inhaltlichen Fragen der Studierenden, aber auch von Fragen zu Fachsprache, Textsorte(n) und Textsortenkonventionen bzw. zur situativen Einbettung des Textes in die entsprechende Handlungskette.

Die Texte, die daraufhin von den Studierenden erstellt wurden, wiesen die gängigsten Merkmale popularisierender Texte sowohl inhaltlich und sprachlich wie auch die Textsortenkonventionen, das Layout und die Makround Mikrostruktur betreffend auf. Sie zeugten von kreativen Arbeitsprozessen, die anscheinend auch Spaß gemacht haben. In der gemeinsamen Evaluierung konnten Textsortenkonventionsverstöße und fachliche, terminologische oder sprachlich-stilistische Ausrutscher im Team erkannt und die Texte optimiert werden. Somit wurde auch Training zur Textoptimierung und Überarbeitung schon bestehender Texte geboten. Auf einen der so entstandenen Texte soll nun exemplarisch näher eingegangen werden.

Der Text ${ }^{2}$ (siehe Abb. im Anhang) zeigt eine deutliche Erarbeitung der Makrostruktur wie auch eine markante layoutmäßige Bearbeitung. Es wurde ein reißerischer, appellativ-metaphorisierender Titel Tod dem Magenteufel gewählt. Das Bakterium wird mit dem Teufel anhaftenden Assoziationen in Verbindung gebracht und somit personifiziert, was eine typische Vorgangsweise bei popularisierenden Texten ist. Weiters wird geschickt ein Bezug zu Personen des öffentlichen Lebens hergestellt ("auch James Bond alias Pierce Brosnan könnte betroffen sein"). Das Stereotyp des Agenten und sozusagen Profikillers von bösen Teufeln wird evoziert. Makrostrukturell ist der Text in unterschiedliche Teiltexte mit intertextuellen Verweisen (z. B. auf den Informationstext "siehe links") gegliedert: Er umfaßt einen zusammenfassenden, einführenden Textteil zu Problemstellung, Abhilfe und Forschungserkenntnissen, der an ein Abstract erinnert und den Bezug zum Leser herstellen bzw. dessen Interesse wecken soll ("Modekrankheit unserer streßbeladenen Zeit"); weiters findet sich ein populärwissenschaftlicher Informationstext, der durch fettgedruckte thematische Schwerpunkte untergliedert ist. Er umfaßt die Problemstellung (aufgrund der Gefahren durch den Helicobacter muß eine Therapie erarbeitet werden), die Beschreibung der 
entsprechenden Studie von Dr. B. mit erklärenden Einschüben (z.B. für Placebo) und die Darstellung der Ergebnisse und Erfolge. Sprachlich wird - passend zur Illustration des Textes mit einem Kämpfer gegen das Böse, der immer siegt - eine Kriegs- bzw. Kampfmetaphorik aufgebaut: "die Götter in Weiß haben dem Helicobacter den Kampf angesagt [...]", die Mediziner konnten "in der entscheidenden Schlacht die Oberhand gewinnen", schließlich "wurde der Magenteufel besiegt" und Erfolg stellte sich ein (die Mediziner "triumphierten über den Magenteufel"). All dies sind typische Strategien der Popularisierung. Bei der Darstellung der Ergebnisse kommt es letztlich zu emotionalisierten Bewertungen, die die Leistung der Wissenschafter hervorheben: "Das, was man nie in diesem Ausmaß zu hoffen gewagt hatte, trat ein: ...". Zu den positiven Ergebnissen kommt aber auch die Erwähnung einer Warnung von Dr. B. vor "unkontrollierter Verabreichung" und der "Gefahr der Resistenzentwicklung". Schließlich wird B. noch direkt zitiert, was die Glaubwürdigkeit und den Anspruch auf Seriosität des Geschilderten untermauern soll.

Zudem umfaßt der Text - nach dem Motto ein Fachmann kommt zu Wort - ein Interview mit einer Fachkapazität als weitere (strategische) Textsorte der Popularisierung. Dieser Teil weist eine typische Interviewstruktur mit meist rhetorischen Fragen auf. Der thematische Verlauf wird durch die Fragestellung gesteuert, Erklärungen werden als "Anm. der Red." eingeführt. Als Illustration dazu wurde ein bekannter ORF-Reporter gewählt. Auch hier steht das Bild für Seriosität und Wissenschaftlichkeit. Fiktive Dialoge haben generell argumentativen, aber auch implizit didaktischen Charakter: "Die in solchen Dialogen vorgebrachten Fragen, Antworten, (Schein)-Argumente und Gegenargumente unterstützen wirkungsvoll das Kommunikationsverfahren Überzeugen, das die eigentliche Kommunikationsabsicht des Aufklärungstextes ist" (Gläser 1990:222). Kienpointner (1983:71) spricht in dieser Hinsicht auch von "konstruierter Argumentation", d.h. "zu Demonstrationszwecken erfundener Argumentation” (ibid.), und Gläser (1990:222) beschreibt diese Diskursstrategie der Popularisierung wie folgt: "Dialogpartner sind Fachleute und Laien, deren Fragen und Meinungen [...] stark verallgemeinert sind. Häufig werden sie mit Porträts von Personen verbunden, die zwar Fotomodelle sind, aber vom Typ her zu dem Inhalt der jeweiligen Äußerung passen". Genau dies haben die Studierenden umgesetzt, als sie das Foto des ORF-Reporters als seriösem Mediziner zur Illustration ihres Textes herangezogen haben. So kann über zuerst unbewußt und spielerisch angewandte Strategien der Popularisierung zu einer systematischen Aufbereitung der Grundcharakteristik popularisierender Texte im Unterricht gelangt werden, denn "eine kontrastive interkulturelle Textsortenkompetenz schließt produktive Kompetenz mit ein" (Schäffner 2002:42).

Im Anschluß an die Evaluierung der entstandenen Texte erfolgte ein Vergleich mit einem schon bestehenden, dem gestellten Arbeitsauftrag entsprechenden Text, wobei sich herausstellte, daß die Studierenden in ihren Versionen die relevanten Textkonventionen und Vertextungsmuster erkannt und auch umgesetzt hatten. Eine solche textsortenspezifische Auseinander- 
setzung mit dem popularisierenden Fachtext und medizinischen Inhalten dient als Vorbereitung zur Bearbeitung einfacher medizinischer (popularisierender) Fachtexte in der allgemeinen Übersetzungsübung. Aufbauend auf dem so erworbenen Grundlagenwissen und einer solchen Textanalyse und kreativen Textarbeit, die die Studierenden motiviert und in ihrer Verantwortung Text und Translat gegenüber, aber auch in ihrem Vertrauen auf ihre eigene Kompetenz und ihr Grundlagenwissen als professionelle TranslatorInnen bestärkt, kann zur Translation popularisierender Texte in Übersetzungsübung und Fachsprache übergegangen werden. Zudem sind die auf diese Weise erworbenen (oder erweiterten) Basiskenntnisse (Textanalyseparameter, Sensibilisierung für Textspezifika und Fachsprachenspezifika und deren Relevanz für die Umsetzung oder Adaptation in der Translation) im Prinzip sprachunabhängig und können in ihren Grundzügen auch auf andere Sprach- und Fachbereiche übertragen werden.

\subsection{Der popularisierende Fachtext in der Fachsprache Medizin}

\subsubsection{Fachsprache Medizin und erster Übersetzungsauftrag}

In LVs zur Fachsprache Medizin eignen sich popularisierende Texte gut als Einstieg bzw. Anknüpfungspunkt zu schon besuchten LVs. So kann auf schon erworbenes Wissen zu Textsorten und deren Charakteristik bzw. Translationstechniken zurückgegriffen werden. Ein wesentliches Erfordernis, das im Rahmen der fachsprachlichen LV hinzukommt, ist der verstärkte Bedarf an gezieltem systematischen Erwerb von Fachwissen und -kenntnissen, was in das didaktische Konzept Eingang finden sollte (cf. Haider 2002:31). (Sprachunabhängiges) fehlendes Sachwissen stellt den Regelstatus zu Beginn der LV dar und sollte auch als Normalsituation deutlich gemacht werden. Dies ist für eine offene Lehr- und Arbeitssituation wichtig (ibid.), bringt jedoch auch den Anspruch an den Lehrenden mit sich, einen den Schritt-für-Schritt-Erwerb von Sachwissen und Kompetenzen ermöglichenden Aufbau der LV und damit eine ebensolche Textprogression bzw. Auswahl von Arbeitsaufträgen zu gewährleisten.

Dem sollte in einer LV zur Fachsprache Medizin die Wahl eines Themas mit dazugehörigem situativem Rahmen, nämlich dem Basisszenario Tätigkeit in einem pharmazeutischen Betrieb, Rechnung tragen. Als Rahmenthema wurden Antibiotika bzw. Antibiotikalehre gewählt. Als Einstieg in die Materie wurde, aufbauend auf der Beschäftigung mit Werbetexten in von den TeilnehmerInnen schon besuchten LVs, über eine (einsprachige) Analyse und einen Vergleich von medizinischen Werbetexten für den Fachmann und dem popularisierenden Werbetext für den Laien ein erster Zugang zur Fachmaterie geschaffen. So wurde eine Wiederholung und Auffrischung der wichtigsten Merkmale von Fachsprache und Fachtext, Popularisierung und popularisierendem Fachtext gewährleistet. Die Abklärung der vor allem in der Fachwerbung vorkommenden Termini und inhaltlichen Fragen führte zu einer ersten Auseinandersetzung mit Lexikon- 
einträgen in Ausgangs- und Zielsprache, einem Vergleich dieser Textsorten und einer entsprechenden ersten Recherche mit diversen Hilfsmitteln wie Hand- und Wörterbüchern, elektronischen Medien, aber auch zu ersten Kontakten mit Gewährspersonen.

Die Abklärung thematischer Fragen hatte die meisten Studierenden unter anderem zu einer Internetrecherche veranlaßt. Sie hatten deutsch-, aber auch französischsprachige Informationstexte aus dem Internet konsultiert, die meist popularisierender Natur waren. Das recherchierte Wissen erwies sich jedoch als recht heterogen, wie auch der Schwerpunkt der Recherche auf muttersprachlichen, also deutschsprachigen Texten, gelegen hatte. Daher wurden die Studierenden nun mit einem ersten Übersetzungsauftrag konfrontiert, in dem sie ihre Vorarbeiten verwerten konnten. Der Auftrag war im Medium Internet angesiedelt und umfaßte die Erstellung eines popularisierenden Textes, zu dem leicht Paralleltexte zu recherchieren waren. Er lautete: Sie arbeiten bei Netdoctor international; ein Informationstext zu Haemophilus influenzae soll mehrsprachig ins Netz gestellt werden, der vorliegende Text soll übersetzt und für das Internet aufbereitet werden. Der vorliegende Text war ein französischsprachiger, einführender Informationstext aus einem französischen Pendant zum österreichischen Netdoctor. Von der inhaltlichen Themenstruktur deckte er sich mit den von den Studierenden recherchierten Informationen, sodaß keine Verstehensprobleme mehr vorlagen und sich die Studierenden auf die Erarbeitung der fremdsprachlichen Terminologie und der Spezifik des français médical popularisant konzentrieren konnten. Die Bearbeitung führte neben der auf der Erfahrung der Studierenden mit Internettexten basierenden translationsrelevanten Auseinandersetzung mit Text und Textsorte auch zu einer die Fachinhalte betreffenden, weiterführenden, teilweise kulturvergleichenden Recherche medizinischer Natur. Über eine den Studierenden bekannte Textsorte und deren Konventionen konnte so der Einstieg in unbekannte, zu erarbeitende Materie erleichtert werden. Dazu bietet die Popularisierung ein wertvolles Forum.

\subsubsection{Vom Internettext zur internen Mitarbeiterschulung: der Trans- lator als Popularisator und Didaktiker bzw. Translator für den Eigenbedarf}

Um die Studierenden mit dem français médical vertraut zu machen und sie zu motivieren, französischsprachige, thematisch spezifische Texte zu konsumieren, d.h. durch eine für den Eigengebrauch bestimmte Translationsarbeit sich selbst zugänglich zu machen, und gleichzeitig Basiswissen zum Thema Antibiotika aufzubauen und dazu zu recherchieren, lautete ein weiterer, der Popularisierung zuzuordnender, Arbeitsauftrag folgendermaßen: Für Schulungsunterlagen im Bereich einer Elsässer Pharmafirma soll zweisprachiges Material zum Thema Antibiotikalehre verfaßt werden. Dazu wurde den Studierenden - neben sonstigen Lehr- und Handbüchern und über Bibliothek und Internet zugänglichem Material - ein französischsprachiges Handbuch 
zur Antibiotikalehre zur Verfügung gestellt. Die Studierenden sollten in Kleingruppen für eine interne Mitarbeiterschulung jeweils eine Kurzfassung eines von ihnen ausgewählten Themenbereiches erstellen und für die Schulung geeignete Schulungsunterlagen (wie Handouts, Overheadfolien....) erarbeiten bzw. sich auch für eventuelle Fragen der zu schulenden Mitarbeiter rüsten. Als Vorbereitung beschäftigten wir uns im Kurs mit der Analyse und exemplarischen Übersetzung von Paralleltexten, d.h. mit Auszügen aus schon bestehenden Schulungsunterlagen. Dadurch bekamen die Studierenden eine Vorstellung von der Textsorte und deren Charakteristik, sie wurden mit Paralleltexten in der Zielsprache konfrontiert und erweiterten über didaktisch aufbereitetes Material ihr Sachwissen zur Materie und Terminologie. Außerdem übten sie sich in der Herübersetzung dieser Texte, die jedoch translatologisch noch keine gravierenden Probleme darstellten, da hohe Parallelität in der sprachvergleichenden Betrachtung der Textsorte gegeben ist - und gegeben sein muß, denkt man an die Internationalität von Pharmafirmen und die Notwendigkeit, die entsprechenden Informationsunterlagen von Lokalisierung - vorerst - freizuhalten. Als kognitiv aufwendige und komplexe Leistung ist hier sicher das Aufbereiten und Sich-selbst-Verfügbarmachen von Sachinformation und anschließender "Selektion und Komprimierung der Informationen" (Göpferich 1996:13) für die Trainerstunde durch die Studierenden zu sehen. Außerdem kann so Fachwissen autonom erworben werden, was erlaubt, sich im Unterricht primär auf translationsrelevante Aspekte zu konzentrieren.

Der Einsatz von massiv didaktisch aufzubereitenden popularisierenden Texten hatte zudem den Effekt, daß sich die Studierenden mit einem fremdsprachigen Text zur Thematik intensiv auseinandersetzten und ihnen fehlendes Sachwissen recherchierten, um dies dann so aufzubereiten, daß Laien daraus einen Wissenszuwachs ziehen konnten. Die Anwendung popularisierender Strategien hatte damit einen dreifachen Effekt: Zum einen eine exakte Aufbereitung im autonomen Lernen für sich selbst, zum anderen eine zweite Verarbeitungsstufe zur didaktisierenden Aufbereitung für andere, und schließlich die gemeinsame Zusammenführung des erworbenen Wissens in der Nachbereitung der internen Mitarbeiterschulung. Dazu kam die kontrastive Auseinandersetzung mit medizinischem Faktenwissen, der entsprechenden Terminologie in Ausgangs- und Zielsprache und die Strukturierung des so erworbenen Wissens für ein (relativ) fachexternes Publikum, wobei die aktive Trainerrolle in der Schulungssimulation sicherlich zur Motivation und zur aktiven Übernahme von Verantwortung im Kursgeschehen beitrug. Die Studierenden hatten für ihre Schulungsteilnehmer teilweise eigene Terminologielisten $\mathrm{zu}$ den bearbeiteten Themenschwerpunkten erarbeitet. Auffallend war, daß generell die Folien zur Präsentation innerhalb der Schulung und die als Handreichung für später gedachten Handouts in Struktur, Umfang und thematischer Gliederung meist unterschiedlich gestaltet waren. Die Studierenden hatten also ganz bewußt je nach Medium, Funktion und Situation auf unterschiedliche Strategien der Didaktisierung und damit auch Popularisierung zurückgegriffen und dabei den entsprechenden Textsortenkonventionen Rechnung getragen. Bei Nord (2002:18) ist 
z.B. auch die Rede von "Background-Texten". Die Arbeit mit Schulungsunterlagen stellte in gewissem Sinne auch eine solche Arbeit mit Background-Texten dar, mit deren Hilfe gezielt Hintergrundinformation verfügbar gemacht werden kann.

\subsubsection{Vom popularisierenden Fachtext zum Fachtext Medizin: die Text- sorte Beipacktext}

Die Textsorte Beipacktext ist in der Literatur gut beschrieben und kann in ihrer Spezifik theoretisch aus der Sekundärliteratur (cf. Gläser 1990; cf. Schuldt 1992) erarbeitet werden. Auf das Szenario Pharmafirma bezogen, muß jedoch eine weitere und für die Studierenden wie auch für die Lehrsituation interessante Differenzierung vorgenommen werden, nämlich in Beipacktexte vor und nach Registratur bzw. Beipacktexte (oder Textbausteine von Beipacktexten), die primär für den ärztlichen Gebrauch, also die Information des Fachmannes verfaßt werden, und Beipacktexte von Medikamenten zur Anwendung durch den Patienten. Erstere weisen einen sehr hohen Fachlichkeitsgrad auf, wohingegen letztere hohen Popularisierungsgrad aufweisen. Es läßt sich somit auch bei der Textsorte Beipacktext, je nach Medikamentenart, je nach Intention der Pharmafirma, der Natur der Interaktionspartner bzw. den pragmatischen Rahmenbedingungen wie freier Erhältlichkeit, Verschreibungspflicht oder nur für den Spitalsgebrauch eingesetzte Medikamente, eine Graduierung von hohem Popularisierungsgrad und niederem Fachlichkeitsgrad bis hin zu niedrigem bis gar keinem Popularisierungsgrad und extremem Fachlichkeitsgrad ausmachen. Diese Graduierung muß dem Translator bewußt sein und wird die Auswahl seiner Strategien determinieren. Die Arbeit mit diesen Textbausteinen und Texten erweist sich aufgrund des hohen Konventionalisierungsgrades und der starken Abstufungen nach Fachlichkeits- bzw. Popularisierungsgrad in bezug auf die Erarbeitung von translationsrelevanten Textsortenkonventionen, den Umgang mit pragmatisch-bedingten Rahmenbedingungen (z.B. der rechtlichen Situation) und nicht zuletzt kultureller Unterschiede als sinnvoll.

\section{Ergebnisse und Schlußfolgerungen}

Was sollen die Studierenden aus der Beschäftigung mit Popularisierung für ihren späteren Berufsalltag nun mitnehmen? Welche Zielsetzung verfolgt der Didaktiker? Durch die Einbeziehung popularisierender Texte in den Translationsunterricht entwickeln die Studierenden ein Einschätzungsvermögen für Fachlichkeit und Fachlichkeitsgrade, lernen Texte hinsichtlich dieser Größen einzuordnen und zu beurteilen und vor allem auch aktiv zu gestalten. Strategien der Popularisierung, die auch ganz allgemeine Kenntnisse über unterschiedliche Kommunikationsverfahren, Argumentationsstrukturen, stilistische Effekte, appellative Strategien u.v.m. vermitteln, 
werden erkannt, angewendet und im Sprach- und Kulturvergleich analysiert und kreativ umgesetzt. Die Studierenden setzen sich intensiv mit einem Text in seiner sprachlichen und außersprachlichen Einbettung auseinander und gewinnen so den Blick für intratextuelle, aber auch inter- und extratextuelle Zusammenhänge. Sie werden zur horizontalen und vertikalen makro- wie auch mikrostrukturellen Grob- und Feinanalyse des Textes angeregt. Außerdem erfahren sich die Studierenden als aktive Gestalter von Texten, die in einem bestimmten Umfeld funktionieren müssen. Neben der textuellen und pragmatischen wird die kulturelle Komponente vor Augen geführt und praktisch umgesetzt. Nebenbei wird nicht zuletzt durch die notwendige intensive Recherchearbeit Fachwissen erworben und erweitert.

Somit lernen Studierende anhand popularisierender Texte bzw. durch die Popularisierung von Texten ihr passiv vorhandenes Textsorten- und Textsortenkonventionswissen zu aktivieren und gezielt einzusetzen. Sie sind gezwungen, sich mit Verstehen und Verständlichkeit verstärkt auseinanderzusetzen und werden angehalten, sich translationsrelevant unabdingbare Kompetenzen $\mathrm{zu}$ erarbeiten und Komponenten wie Kulturtransfer und Lokalisierung oder auch Internationalisierung (cf. Göpferich 1997:157) aktiv als Texter anzuwenden, um sie dann auch als Translator erkennen, einsetzen und anwenden zu können. Zudem fördert die Arbeit als Popularisator die Ausrichtung auf den Text als komplexe Einheit-in-Funktion und -inSituation. Die Studierenden entwickeln ein (Problem)Bewußtsein für zweckgerichtete Textproduktion und haben so ein funktionierendes Bindeglied im Curriculum von der allgemeinsprachlichen hin zur fachsprachlichen Übersetzung in den unterschiedlichen Graduierungsstufen von Popularisierung und Fachlichkeit. Popularisierende (Fach)texte können daher in der Ausbildung von TranslatorInnen sowohl zum Training in der rezeptiven und produktiven (kreativen) Textarbeit herangezogen werden, als auch zum Aufzeigen kulturspezifischer Elemente, wie auch zum Einarbeiten in die fachliche Materie. Dies betrifft nicht nur den Bereich der Medizin. Ebenso können popularisierende Texte als didaktisches (Teil)Element zum systematischen Fachwissenaufbau herangezogen werden. Das Popularisieren fördert allgemein die Vertextungskompetenz, schafft aber auch durch die Rezeption, Analyse und Produktion von Texten eine vorbereitende Basis zum Erwerb fachbezogener Translationskompetenzen.

\section{Evaluierung: (Fach)Textlinguistik, translatorische Kompetenz und Translationsdidaktik}

Das heterogene Anforderungsprofil des Translators erfordert heute nicht nur textrezeptive und -reproduktive Kompetenzen, sondern vor allem Kompetenz im situations- und kulturadäquaten Informationsmanagement und somit in der Textneuproduktion. Der Translator ist also auch als Texter für die Genese neuer Texte verantwortlich, die oft in einem zum Ausgangstext unterschiedlichen situativen, kulturellen und pragmatischen Umfeld zu funktionieren haben. Text- bzw. Vertextungskompetenz werden somit zu einer 
Schlüsselkategorie für translatorische Kompetenz - und damit auch für die Translationsdidaktik. Wird Fachlichkeit als ein spezifisches Kennzeichen von Texten-in-Situation und -in-Kultur betrachtet und Fachkommunikation als spezifische komplexe Interaktionsform gesehen, die über sprachliche Parameter weit hinausgeht, so stellt (Fach)Textlinguistik unter dem Postulat eines funktions- und skoposorientierten translatorischen Handelns auch eine relevante Komponente für die Translationswissenschaft dar. In der konkreten Umsetzung von intersprachlicher und inter- und transkultureller Fachkommunikation kann sich daraus eine fruchtbare Interdependenz und Interaktion zwischen (Fach)Textlinguistik und Translationswissenschaft ergeben. Fachtexte haben "die Funktion, eine eindeutige effektive und situativ adäquate Kommunikation über fachliche Gegenstände zu gewährleisten" (Gläser 1990:6). Sie haben sowohl referentiellen wie auch sozialen und interpersonnellen Anforderungen im kommunikativen Umfeld zu genügen, wobei erst die Berücksichtigung situativ-interaktiver, pragmatischer und kultureller Faktoren es ermöglicht, den "kommunikativen Zweck von Fachtexten, also ihre Textfunktion, genauer anzugeben” (Möhn \& Pelka 1984:56). Gerade dies sind für den Translator - und damit auch für die Ausbildung von TranslatorInnen - relevante Faktoren. Die angehenden TranslatorInnen müssen einen Zugang zur Charakteristik von Fachsprachen und Fachtexten bzw. zum Variantenreichtum von Texten unterschiedlichen Fachlichkeitsgrades und in unterschiedlicher Funktion und Situation bekommen, aber auch zu deren Bedeutung und Stellenwert bzw. Vorkommensformen in Interaktion und Interaktionsmanagement in verschiedenen Gesellschaftsbereichen. Dies ist vor dem Hintergrund der Globalisierung um so wichtiger, als "une culture ne peut pas se désintéresser de l'évolution scientifique: elle doit l'adopter, l'adapter, se l'approprier, et pour cela guider les changements de son propre langage scientifique" (Sournia 1982:149). Diese Möglichkeit eröffnen popularisierende Texte in der Ausbildung für TranslatorInnen. In der entsprechenden Didaktik sollten intra- und intersprachliche bzw. -kulturelle Textrezeptions- und -produktionskompetenz einen zentralen Stellenwert einnehmen. Textsorten der Popularisierung sind

für die konkrete Umsetzung eines solchen Didaktikanspruches u. E. in hohem Maße geeignet. Lehrende können hier leicht nach Bedarf Akzente setzen; im Grunde sind popularisierende Texte sogar anspruchsvoller als reine Fachtexte, da sie über die fachliche und terminologische Komponente hinaus auf allen Ebenen der Textualität sowie in ihrer unterschiedlichen, interaktanten- und zweckbedingten Funktionalität hohe Anforderungen an ein holistisches translatorisches Arbeiten stellen. - Und ein Bewußtsein für diese holistische Komponente translatorischen Arbeitens bei den Studierenden zu wecken, ist eine Aufgabe der Translationsdidaktik unter vielen.

\section{Bibliographie}

Albin, Verónica (1998). "Translating and Formatting Medical Texts for Patients with Low Literacy Skills.” Henry Fischbach (Hrsg.) (1992). Translation and 
Medicine. American Translators Association Scholarly Monograph Series; X. Amsterdam/Philadelphia: Benjamins, 117-129.

Authier, J. (1982). "La mise en scène de la communication dans des discours de vulgarisation scientifique." Langue française 53, 34-47.

Feyrer, Cornelia (2002). "Le document de visite: les mérites d'une sorte de texte pour la didactique de traduction en langue de spécialité." LSP \& Professional Communication 2(1), 67-83.

Feyrer, Cornelia \& Peter Holzer (Hrsg.) (2002). Translation: Didaktik im Kontext. InnTrans. Innsbrucker Beiträge zu Sprache, Kultur und Translation; 1. Frankfurt am Main: Lang.

Gläser, Rosemarie (1990). Fachtextsorten im Englischen. FFF; 13. Tübingen: Narr. Göpferich, Susanne (1995). Textsorten in Naturwissenschaften und Technik. Pragmatische Typologie-Kontrastierung-Translation. FFF; 27. Tübingen: Narr.

Göpferich, Susanne (1996). "Textsortenkanon: Zur Text(sorten)auswahl für fachsprachliche Übersetzungsübungen." Andreas F. Kelletat (Hrsg.) (1996). Übersetzerische Kompetenz. Beiträge zur universitären Übersetzerausbildung in Deutschland und Skandinavien. Frankfurt am Main: Lang, 9-38.

Göpferich, Susanne (1997). "Der Kommunikationsmittler im Wissenstransfer vom Fachmann zum Laien: Neue Aufgaben in der Übersetzerausbildung." Horst W. Drescher (Hrsg.) (1997). Transfer. Übersetzen-Dolmetschen-Interkulturalität. Frankfurt am Main: Lang, 153-174.

Göpferich, Susanne (2002). "Vom Übersetzen und der Technischen Redaktion zur Interkulturellen Technischen Redaktion." Feyrer \& Holzer (2002), 105-121.

Haider, Sabine (2002). "Aspekte der Fachsprachendidaktik: Kontrastiver Fachwissenerwerb und inhaltsseitige Terminologiearbeit als Grundlagen der Qualitätssicherung.” Feyrer \& Holzer (2002), 29-38.

Hoffmann, Lothar ('1985). Kommunikationsmittel Fachsprache. Eine Einführung. FFF; 1. Tübingen: Narr.

Jacobi, Daniel (1984). "Du discours scientifique, de sa reformulation et de quelques usages sociaux de la science." Langue française 64, 37-52.

Kienpointner, Manfred (1983). Argumentationsanalyse. Innsbrucker Beiträge zur Kulturwissenschaft; Sonderheft 56. Innsbruck: Verlag des Instituts für Sprachwissenschaft der Universität Innsbruck.

Kromann, Hans-Peder \& Knud Troels Thomsen (1989). "Akzente der Fachsprachenforschung von heute und morgen. Bericht vom Kopenhagener Werkstattengespräch 1.-2. Juni 1988." Terminologie et traduction 1, 137-160.

Löning, Petra (1994). "Versprachlichung von Wissensstrukturen bei Patienten." Angelika Redder \& Ingrid Wiese (Hrsg) (1994). Medizinische Kommunikation. Diskurspraxis, Diskursethik, Diskursanalyse. Opladen: Westdeutscher Verlag, 97-114.

Möhn, Dieter \& Roland Pelka (1984). Fachsprachen. Eine Einführung. Germanistische Arbeitshefte; 30. Tübingen: Niemeyer.

Nord, Christiane (2002). "Anleitung zum Trockenschwimmen - Übersetzungspropädeutik als Vorstufe zum Übersetzungsunterricht.” Feyrer \& Holzer (2002), $15-28$.

Pöckl, Wolfgang (1990). "Französische Fachsprachen.” Günter Holtus, Michael Metzeltin \& Christian Schmitt (Hrsg.) (1990). Lexikon der Romanistischen Linguistik (LRL) V/1. Le français. Tübingen: Niemeyer, 267-282.

Schäffner, Christina (2002). "Entwicklung von übersetzungsorientierter Textkompetenz." Feyrer \& Holzer (2002), 41-58.

Schuldt, Janina (1992). Den Patienten informieren. Beipackzettel von Medikamenten. FFF; 15. Tübingen: Narr. 
Sournia, J. C. (1982). "Culture et langage scientifique." La banque des mots 24, 141-149.

Wiese, Ingrid (1984). Fachsprache Medizin. Eine linguistische Analyse. Linguistische Studien. Leipzig: VEB Verlag Enzyklopädie.

${ }^{1}$ Die von Göpferich (1995:68) vorgeschlagene Differenzierung zwischen popularisierend und populärwissenschaftlich wird hier nicht berücksichtigt, da die herangezogenen Texte meist - mit einer gewissen Graduierung - Charakteristika beider Gruppen aufweisen; wohl aber ist eine Differenzierung zwischen dem Translator eines popularisierenden Textes und dem Translator als Popularisator für unsere Zwecke relevant.

${ }^{2}$ Frau Cosima Hufler sei an dieser Stelle sehr herzlich für ihre Bereitschaft, ihren Text für diesen Beitrag zur Verfügung zu stellen, gedankt. 


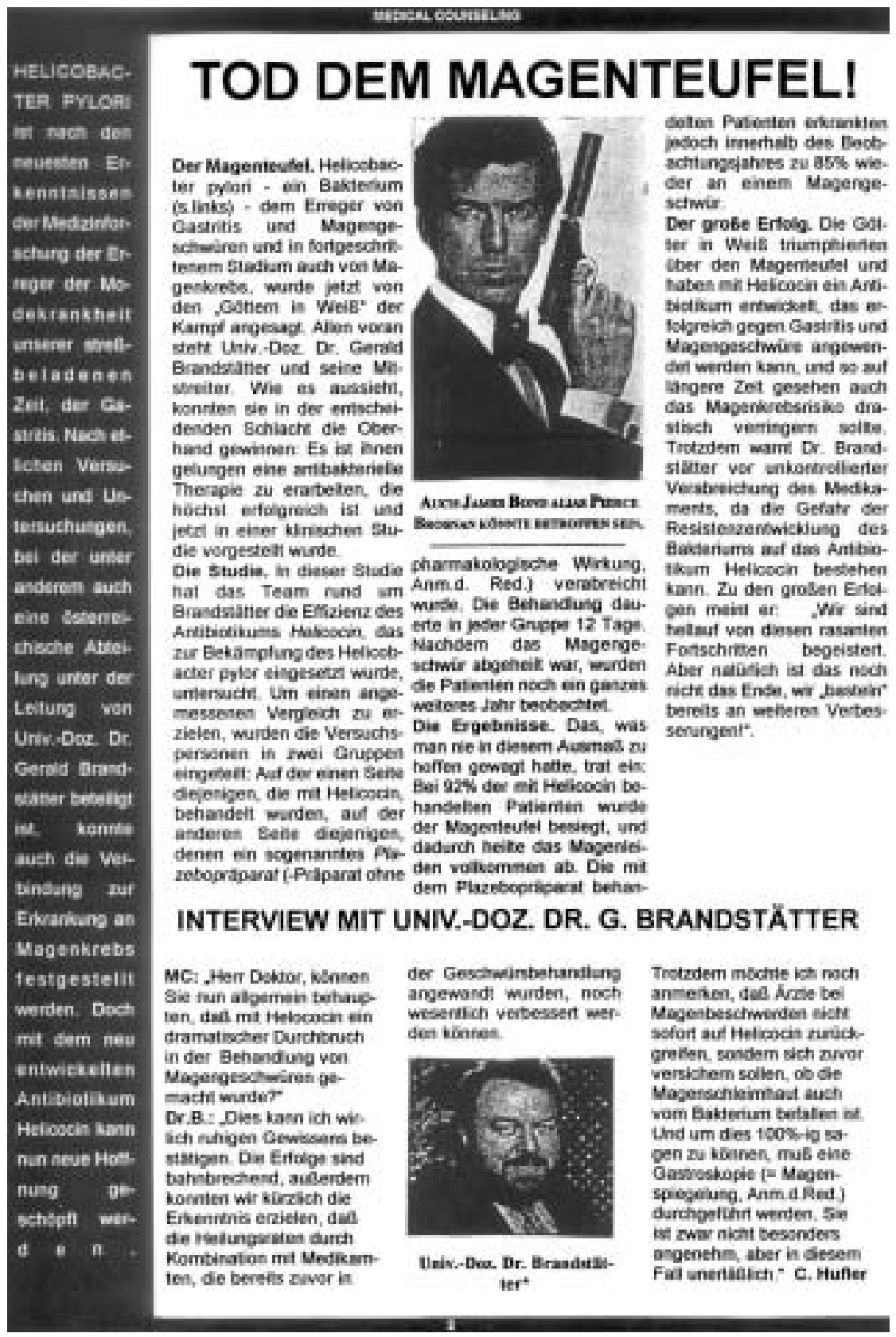

